\title{
Automatic Valve-Rejection Algorithm for Cardiac Doppler Ultrasound Systems
}

\author{
Tatsuro Baba, ${ }^{1}$ Shuichi Adachi, ${ }^{2}$ and Masatsugu Taiko ${ }^{2}$ \\ ${ }^{1}$ Toshiba Medical Systems Corporation, 1920-2, Usuba, Otawara, Tochigi 3240035, Japan \\ ${ }^{2}$ Keio University, 3-14-1 Hiyoshi, Kohoku-ku, Yokohama 2238522, Japan \\ Correspondence should be addressed to Shuichi Adachi; adachi@appi.keio.ac.jp
}

Received 7 February 2013; Accepted 26 February 2013

Academic Editors: R. Guldiken and B. Tomanek

Copyright (c) 2013 Tatsuro Baba et al. This is an open access article distributed under the Creative Commons Attribution License, which permits unrestricted use, distribution, and reproduction in any medium, provided the original work is properly cited.

\begin{abstract}
In recent years, blood flow diagnosis using Doppler ultrasound systems has become popular. Using these systems, the peak velocity of blood flow is automatically traced. However, because valve signals are mixed with the blood flow signals in a heart chamber, automatic measurements of blood flow are not correctly recorded. To solve this problem, we developed a novel method that adopted system identification. We applied a mathematical model with an electrocardiographic waveform as the input and a trace waveform of the peak velocity as the output. Several mathematical models with different structures and orders were compared to select the optimal model. Using this model, we developed a system that could automatically eliminate the valve signal. We also evaluated our valve-rejection algorithm using simulations based on actual clinical data.
\end{abstract}

\section{Introduction}

Doppler ultrasound systems are widely used for the diagnoses of the heart, abdomen, and other organs and systems. Because blood flow measurement is very complicated, methods for automatic measurements have been developed [1-4]. For example, in the carotid artery, blood flow volume, peak velocity, pulsatility, and so forth. are measured automatically. However, in the heart, because valve motions overlap blood flow, automatic measurements become difficult. Blood flow measurements, such as left ventricular outflow, have been made by manual procedures due to the influence of the aortic valve $[5,6]$. We developed a method for automatic left ventricular outflow measurements and verified its performance using simulations.

\section{Trends and Problems with Blood Flow Measurements}

A Doppler ultrasound diagnostic image for left ventricular outflow is shown in Figure 1. The upper part of the figure shows the ultrasound echo image (B-mode image). The Doppler range gate was set at the tip of the aortic valve, and the blood flow information at this point is shown in the lower part of this figure as a Doppler spectrum image. In this image, the horizontal axis represents time, whereas the vertical axis represents blood flow velocity that corresponds to the Doppler shift frequency. The Doppler spectrum image expresses the time-velocity distribution of blood flow. In addition, the Doppler ultrasound system automatically traces the peak velocity waveform $V_{p}$, which is superimposed as a bright yellow line.

There are two noise sources that affect cardiac blood flow measurements: cardiac wall with a strong, low velocity signal and a valve with a strong, high velocity signal. Although the former seldom influences the $V_{p}$ waveform because of its low velocity, the latter has a significant influence on automatic tracing. For example, an aortic valve signal is usually mixed in a left ventricular outflow. Thus, users manually compensate the $V_{p}$ waveform on the basis of the Doppler spectrum image.

In Figure 1, an electrocardiogram (ECG) waveform is displayed (green line) simultaneously with the $V_{p}$ waveform. Also the R-triggers (sharp peaks) of the ECG waveform are at $-1.48 \mathrm{~s}$ and $-0.65 \mathrm{~s}$. The systolic phase is about $300 \mathrm{~ms}$ from the R-triggers of the ECG waveform. A conventional manual tracing line (from -1.5 to $-1.1 \mathrm{~s}$ ) is displayed in bold cyan. Using this line, medical doctors can estimate left ventricular outflow. 


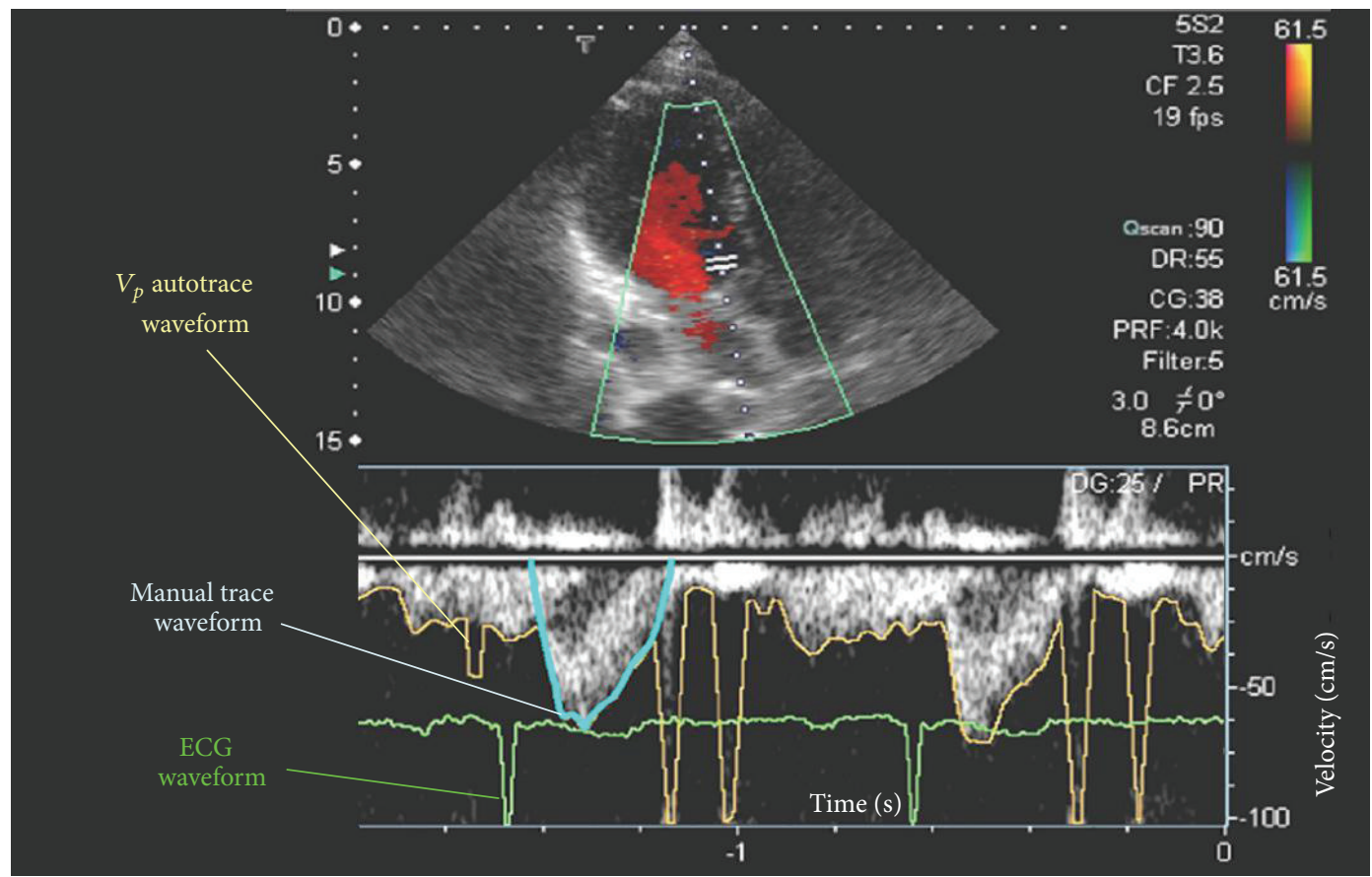

FIGURE 1: Doppler ultrasound image for left ventricular outflow.

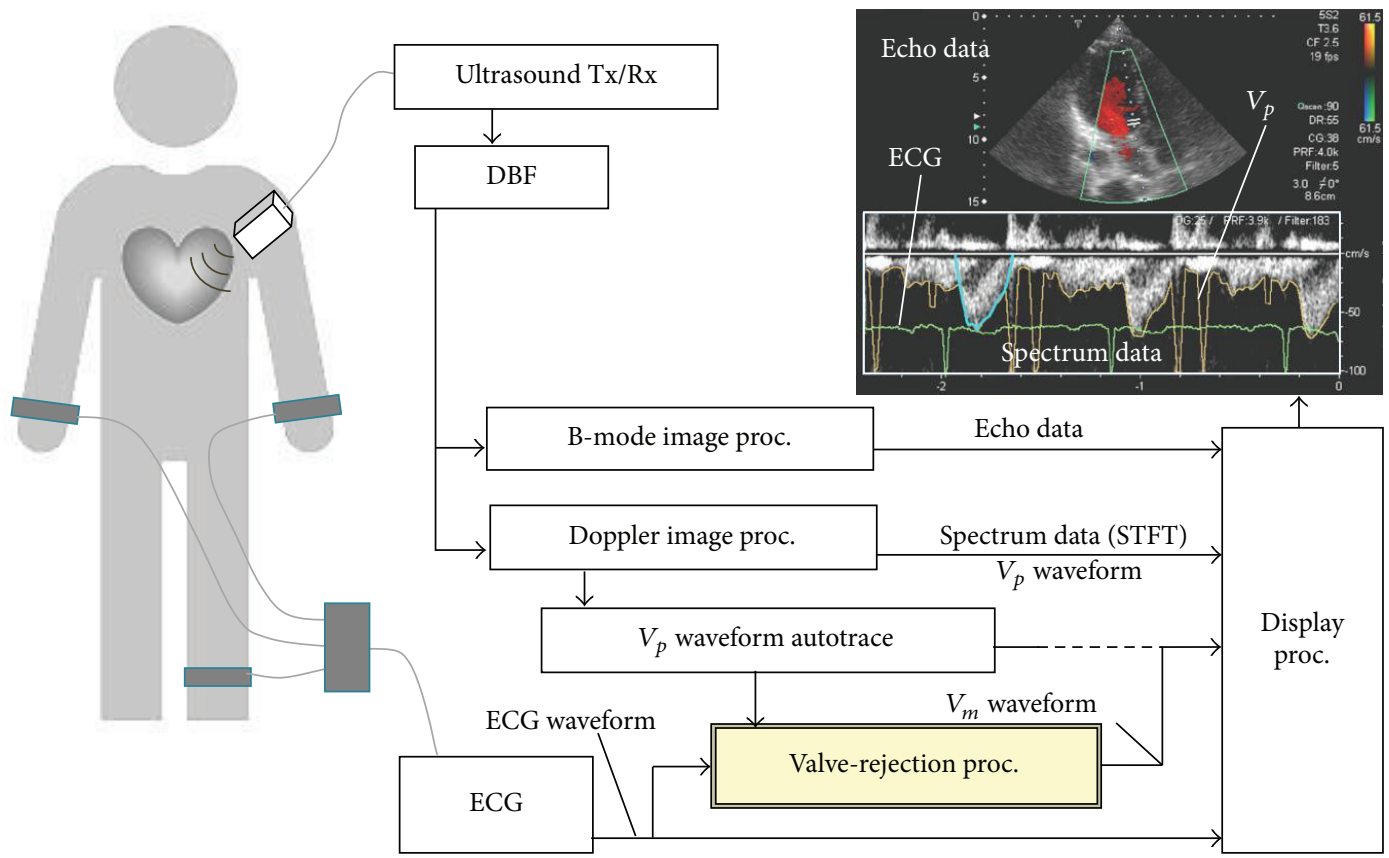

FIGURE 2: Doppler ultrasound system and automatic blood flow measurement.

\section{New Method for Cardiac Valve Rejection}

3.1. Doppler Ultrasound System and Blood Flow Measurements. Figure 2 shows a block diagram for a Doppler ultrasound system and automatic blood flow measurements. The transmitted and received ultrasound signals are processed by a digital beam former (DBF) and are combined as beambased echo data. Based on the ultrasound scan mode, echo data are sequentially distributed for B-mode and Doppler spectrum image processing. The $V_{p}$ waveform is automatically traced and superimposed on the Doppler spectrum image.

Various blood flow measurements that combine the $V_{p}$ and ECG waveforms are known in clinical applications [6]. In an ultrasound system, a B-mode and Doppler spectrum images and the $V_{p}$ and ECG waveforms are simultaneously 


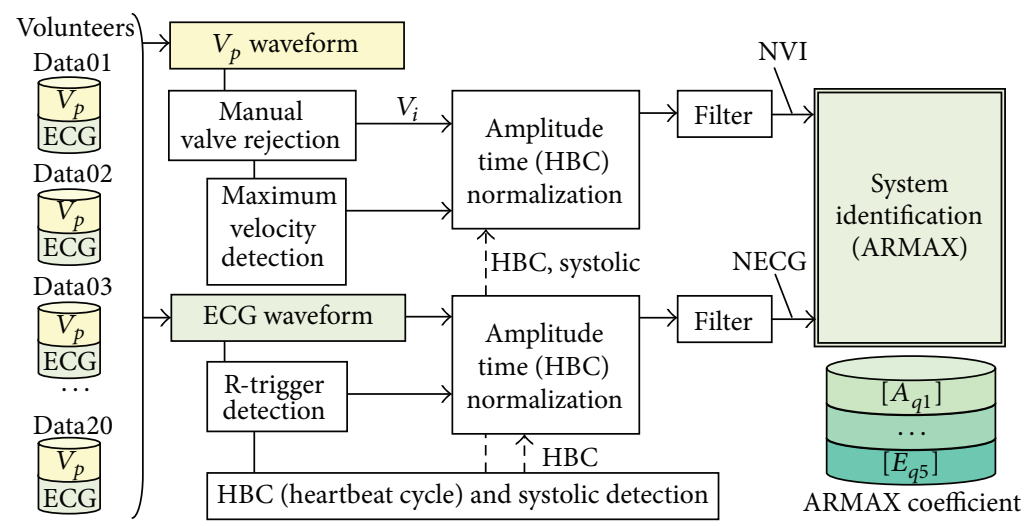

Figure 3: System identification model.

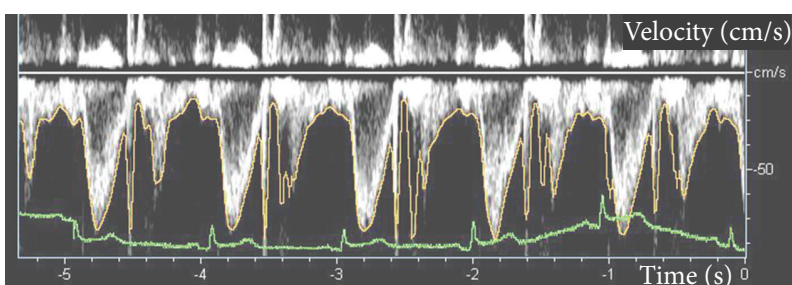

(a)

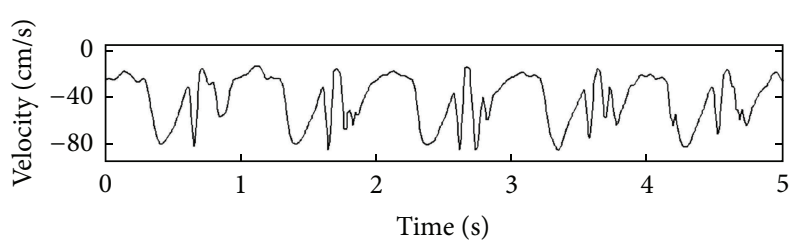

(c)

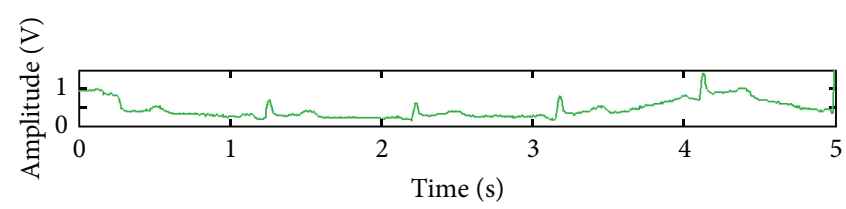

(b)

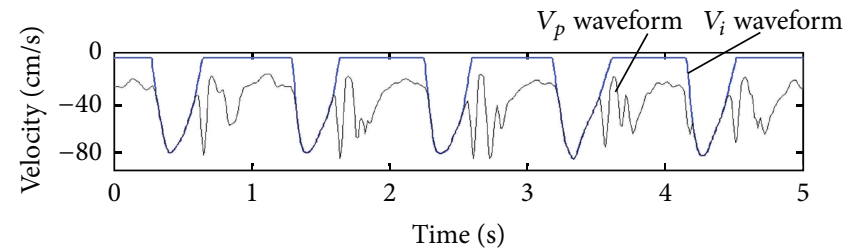

(d)

FIGURE 4: Input and output for the system identification model. (a) Doppler diagnostic image, (b) ECG, (c) $V_{p}$, and (d) $V_{p}$ and $V_{i}$ waveforms.

displayed. Various blood flow measurements are made on the basis of this displayed information.

3.2. System Identification. We identified the system using the data acquired from numerous volunteers, such as $V_{p}$ and ECG waveforms. The system identification model is shown in Figure 3. A $V_{p}$ waveform includes both valve and blood flow information. In an ideal blood flow waveform $\left(V_{i}\right)$, the valve component is manually removed. Because there were variations in the data sets used, the heartbeat cycles and maximum flow velocities were normalized for each data set.

The amplitude of the $V_{i}$ waveform was normalized by the maximum flow velocity of the systolic phase, whereas that of the ECG waveform was normalized by the peak voltage of an R-trigger. A sampling period was normalized by each cardiac cycle and was fixed to 60 (approximately $60 \mathrm{~Hz}$ ). After normalization, a low-pass filter with a onethird cutoff of the sampling frequency (approximately $20 \mathrm{~Hz}$ ) was applied to reject any unnecessary frequency components. After these processing steps, a normalized $V_{i}$ waveform (NVI) and a normalized ECG waveform (NECG) were obtained. The system identification model with NVI and NECG as inputs was constituted, and the coefficient sequences of the mathematical model were calculated.

A diagnostic image obtained with a Doppler ultrasound system is shown in Figure 4(a). The $V_{p}$ and ECG waveforms are shown along with the Doppler image for left ventricular outflow. The ECG and $V_{p}$ waveforms are shown in Figures 4(b) and 4(c), respectively. The ECG waveform was used as an exogenous input, whereas the $V_{i}$ waveform was used as an output. The $V_{p}$ and $V_{i}$ waveforms, from which the aortic valve signal was manually rejected, are shown in Figure 4(d).

Next, we developed the valve-rejection algorithm using the coefficient sequences obtained from the system identification model. A system prediction block diagram is shown in Figure 5. The $V_{p}$ and ECG waveforms are normalized and filtered. The mathematical model generated $V_{e}$, a prediction waveform for $V_{p}$. The blending times for $V_{p}$ and $V_{e}$ were calculated from the difference between NVP and $V_{e}$. The blending weights were changed by blending times. Blending was controlled so that NVP becomes predominant during a 


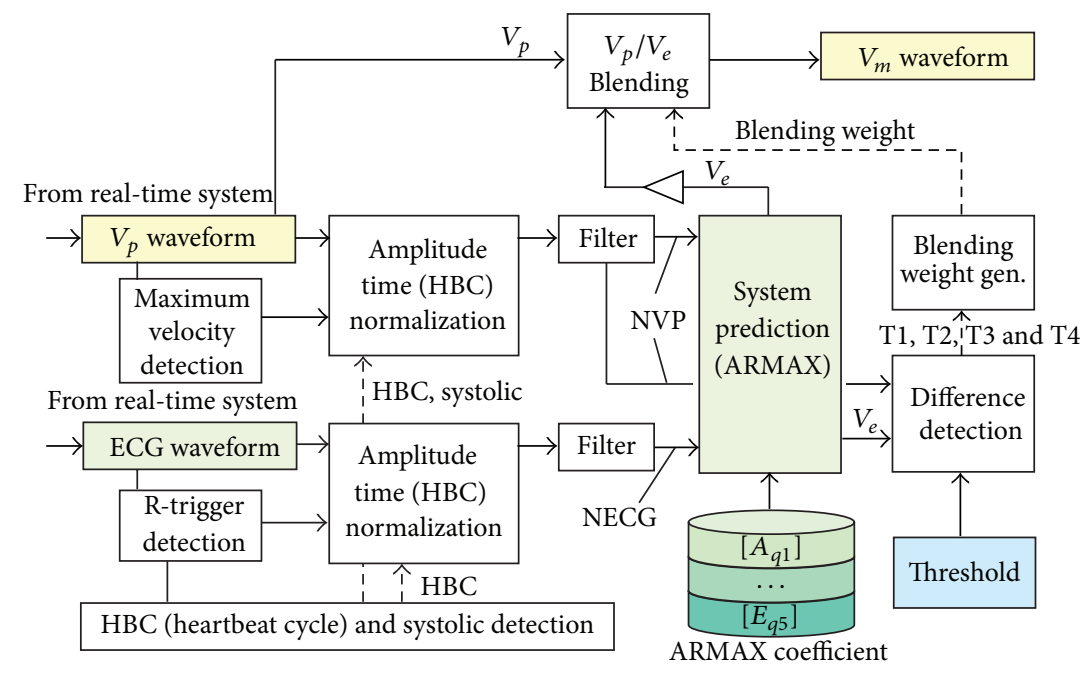

FIGURE 5: System prediction block diagram.

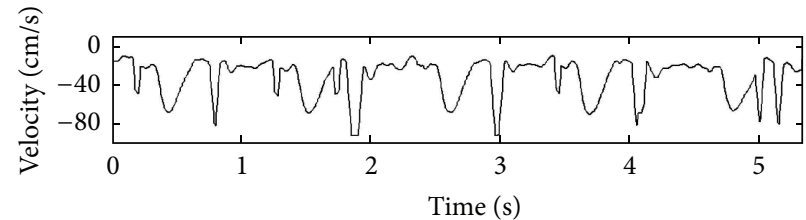

(a)

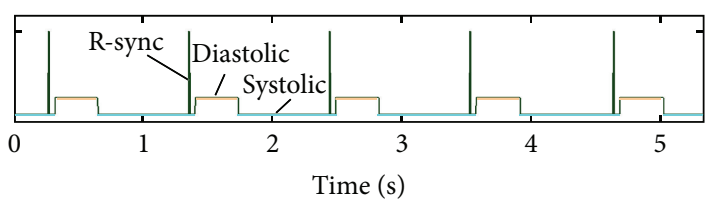

(c)

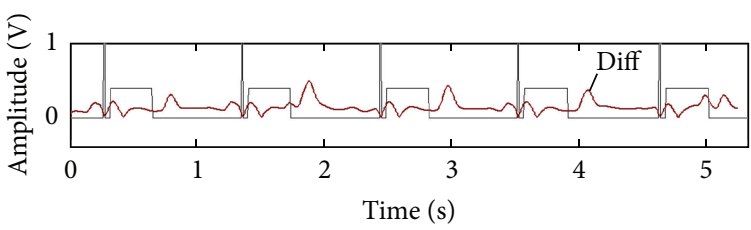

(e)

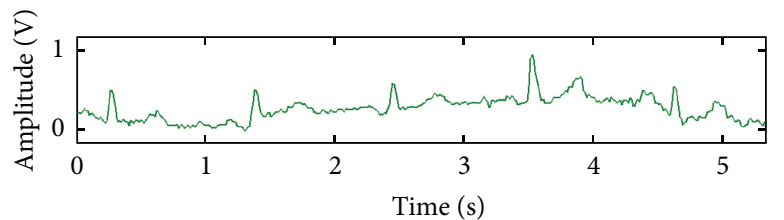

(b)

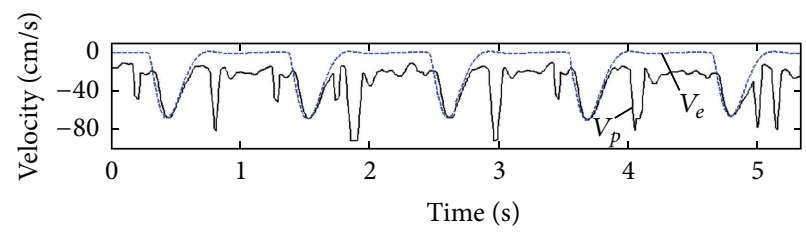

(d)

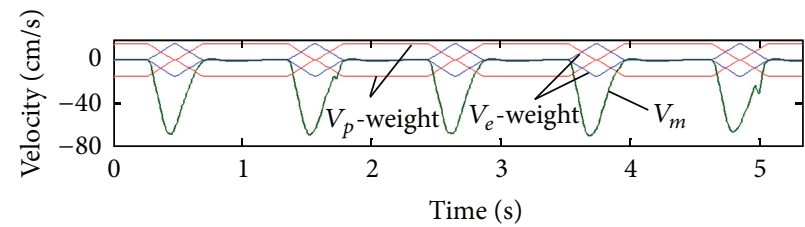

(f)

FIGURE 6: Signal processing for system prediction. (a) $V_{p}$, (b) ECG, (c) complex cardiac cycle, (d) $V_{p}$ and $V_{e}$ waveforms, (e) difference between $V_{p}$ and $V_{e}$ waveforms, and (f) $V_{m}$ waveform.

systolic phase, and an ideal prediction waveform $\left(V_{m}\right)$ was generated.

The signal processing waveforms in Figure 5 are shown in Figure 6. Figures 6(a) and 6(b) show the $V_{p}$ and ECG waveforms, respectively. Figure 6(c) shows the complex cardiaccycle waveform based on the ECG waveform. This was generated from the R-trigger of the ECG waveform and the systolic and diastolic phases. And this can separate each phase by its amplitude level. Figure 6(d) shows the $V_{p}$ and $V_{e}$ waveforms, and Diff waveform of Figure 6(e) shows the absolute difference between these waveforms. Weighting functions for blending were generated on the basis of this difference (Figure 6(e)) and the complex cardiac-cycle waveform (Figure 6(c)). Figure 6(f) shows these weighting functions, $V_{p}$-weight and $V_{e}$-weight, and $V_{m}$ derived from the $V_{p}$ and $V_{e}$ waveforms.

\section{System Optimization}

4.1. Mathematical Model. Parametric models such as ARMAX were used as mathematical models [7]. A parametric model is described as follows:

$$
A(z) \cdot y(z)=\frac{B(z)}{C(z)} \cdot u(z)+\frac{D(z)}{E(z)} \cdot w(z) .
$$




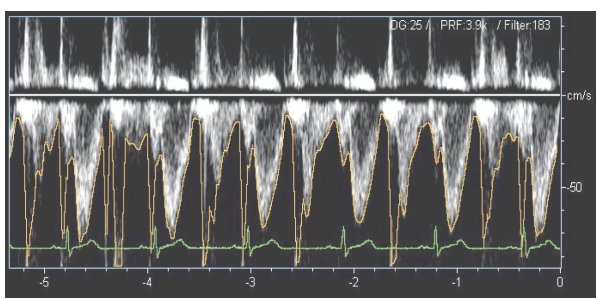

(a)

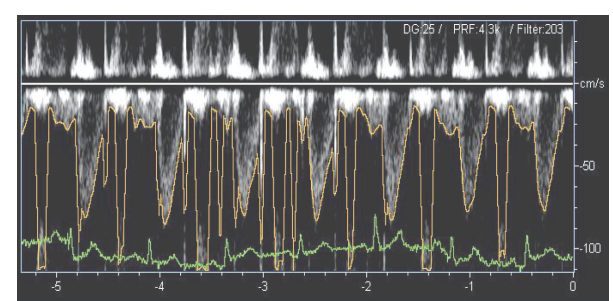

(b)

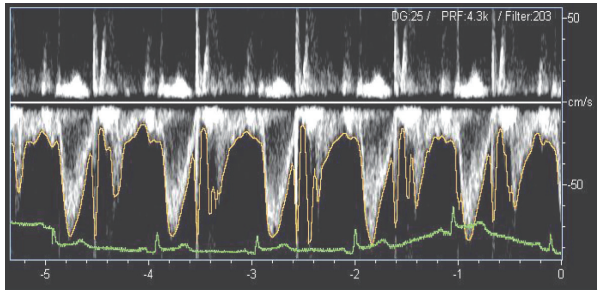

(c)

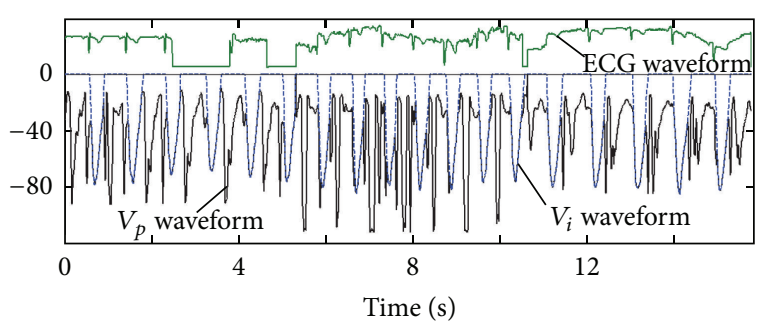

(d)

Figure 7: Data for system identification. Data from volunteer (a) A, (b) B, and (c) C and (d) combined data for system identification.

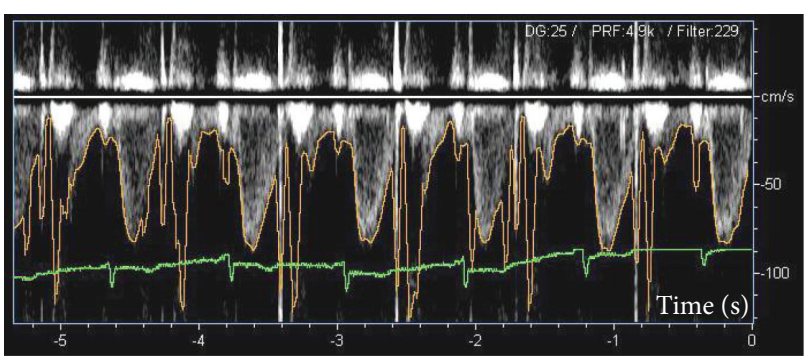

(a)

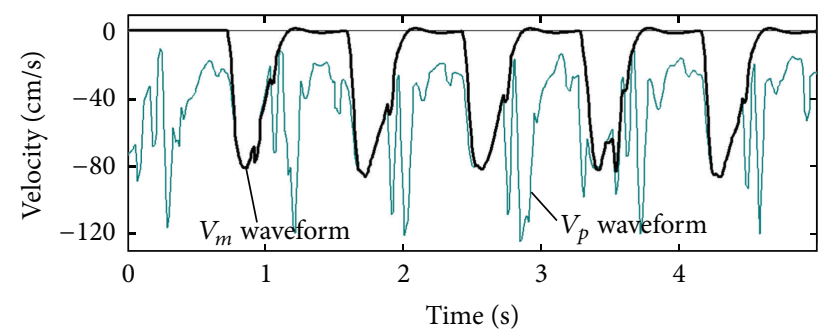

(b)

FIGURE 8: Simulation results for automatically tracing the left ventricular outflow trace. (a) Data from volunteer D and (b) $V_{m}$ waveform (automatic valve-rejection algorithm).

Here, $y(z)$ is output, $u(z)$ is input, and $w(z)$ is white noise. $A(z), B(z), C(z), D(z)$, and $E(z)$ are the coefficient sequences. For the system identification shown in Figure 4, the $V_{p}$ and ECG waveforms were used as $y(z)$ and $u(z)$, respectively. We investigated several models that had different structures and orders.

4.2. Data Used for Modeling. We acquired clinical data using a Doppler ultrasound system. The clinical target was left
TABLE 1: Combined volunteers' data.

\begin{tabular}{llcc}
\hline Data sets & Data A & Data B & Data C \\
\hline Blood flow sensitivity & Middle & Low & High \\
Cardiac cycle & Middle & Fast & Slow \\
Waveform (damping) & Middle & Short & Long \\
\hline
\end{tabular}

ventricular outflow. Because this outflow markedly varies with respect to individual differences, we acquired data from numerous volunteers and combined them. Typical data were chosen from three of 20 volunteers, which had different cardiac cycles, blood flow sensitivities, and waveforms.

The combined volunteers' data are shown in Table 1. Figures 7(a), 7(b), and 7(c) show the left ventricular outflows for volunteers $\mathrm{A}, \mathrm{B}$, and $\mathrm{C}$, respectively. The sampling rate for both $V_{p}$ and ECG waveforms was $120 \mathrm{~Hz}$. The data for 16 heartbeats that combined these waveforms were used for simulations (shown in Figure 7(d)).

4.3. Mathematical Model Evaluation. We evaluated several mathematical models for system identification. After optimizing the model order, data fits to each model were evaluated using the root mean square (RMS) error. Table 2 shows the RMS error for each model; the RMS error for the output error (OE) model was the smallest. The OE model was judged suitable for our valve-rejection algorithm. Table 2 shows the relative RMS errors based on the OE model.

\section{Considerations and Verification}

The OE model was selected as the optimal mathematical model for our study. Furthermore, it was necessary to confirm its performance in the valve-rejection algorithm using additional data other than the simulation data. A 
TABLE 2: Relative RMS errors for the mathematical models.

\begin{tabular}{lcc}
\hline Model & Order & Normalized RMS \\
\hline ARX & 9 & 1.043 \\
OE & 3 & 1.000 \\
ARMAX & 6 & 1.032 \\
BJ & 5 & 1.025 \\
\hline
\end{tabular}

different volunteer's data ((data D) shown in Figure 8(a)) was evaluated.

Figure 8(b) shows the simulation results for the valverejection algorithm. During the first, second, third, and fifth cardiac cycles, $V_{m}$ traced nearly the ideal systolic blood outflow and automatically rejected valve signals. However, during the fourth cardiac cycle, the valve signal was not sufficiently rejected. Although some problems related to robustness were still prevalent, we could confirm the potential of this algorithm.

\section{Conclusions}

Automatic ventricular blood flow measurements using a Doppler ultrasound system are difficult because of valve signal mixing. Here, we developed an automatic valverejection algorithm for left ventricular outflow based on a system identification method. The mathematical model for system identification used an ECG waveform as an exogenous input and a Doppler waveform as an output. We evaluated several different structure models using the clinical data from volunteers and selected the OE model, since it showed optimal performance. In the future, we will improve the stability of this approach and expand its diagnostic applications for all cardiac evaluations.

\section{References}

[1] J. Tschirren, R. M. Lauer, and M. Sonka, "Automated analysis of Doppler ultrasound velocity flow diagrams," IEEE Transactions on Medical Imaging, vol. 20, no. 12, pp. 1422-1425, 2001.

[2] A. Walker, E. Olsson, B. Wranne, I. Ringqvist, and P. Ask, "Accuracy of spectral Doppler flow and tissue velocity measurements in ultrasound systems," Ultrasound in Medicine and Biology, vol. 30, no. 1, pp. 127-132, 2004.

[3] T. Baba, "Investigation of gain optimization technique in Doppler ultrasound system," Acoustical Science and Technology, vol. 30, no. 2, pp. 67-71, 2009.

[4] T. Baba, N. Ohmae, and K. Osuka, "The optimization of ultrasound system Doppler velocity range using hybrid control," Transactions of the Society of Instrument and Control Engineers, vol. 44, no. 9, pp. 760-765, 2008.

[5] J. Lisauskas, J. Singh, M. Courtois, and S. J. Kovács, “The relation of the peak Doppler E-wave to peak mitral annulus velocity ratio to diastolic function," Ultrasound in Medicine and Biology, vol. 27, no. 4, pp. 499-507, 2001.

[6] The Japan Society of Ultrasonics in Medicine, "Standard measurement of cardiac function indexes," Journal of Medical Ultrasonics, vol. 33, no. 2, pp. 123-127, 2006.

[7] S. Adachi, Advanced Level System Identification for Control By MATLAB, Tokyo Denki University Press, Tokyo, Japan, 1996. 

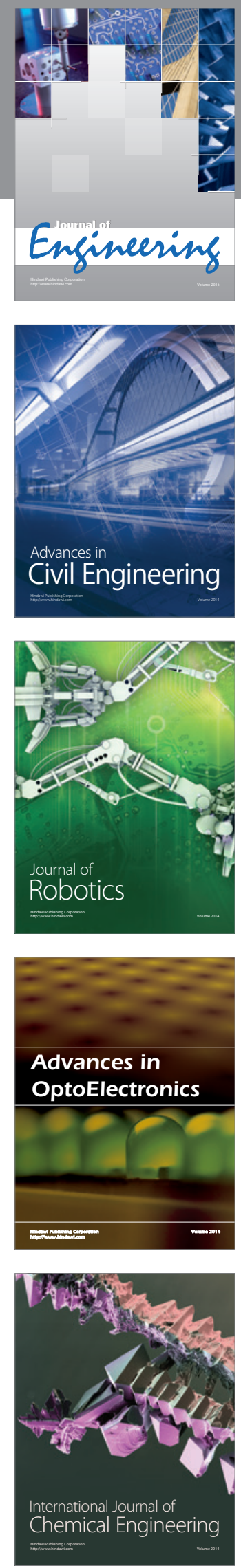

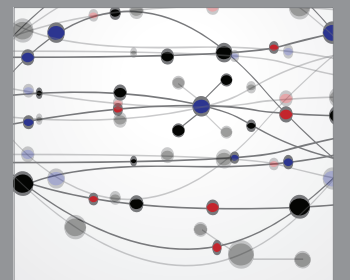

The Scientific World Journal
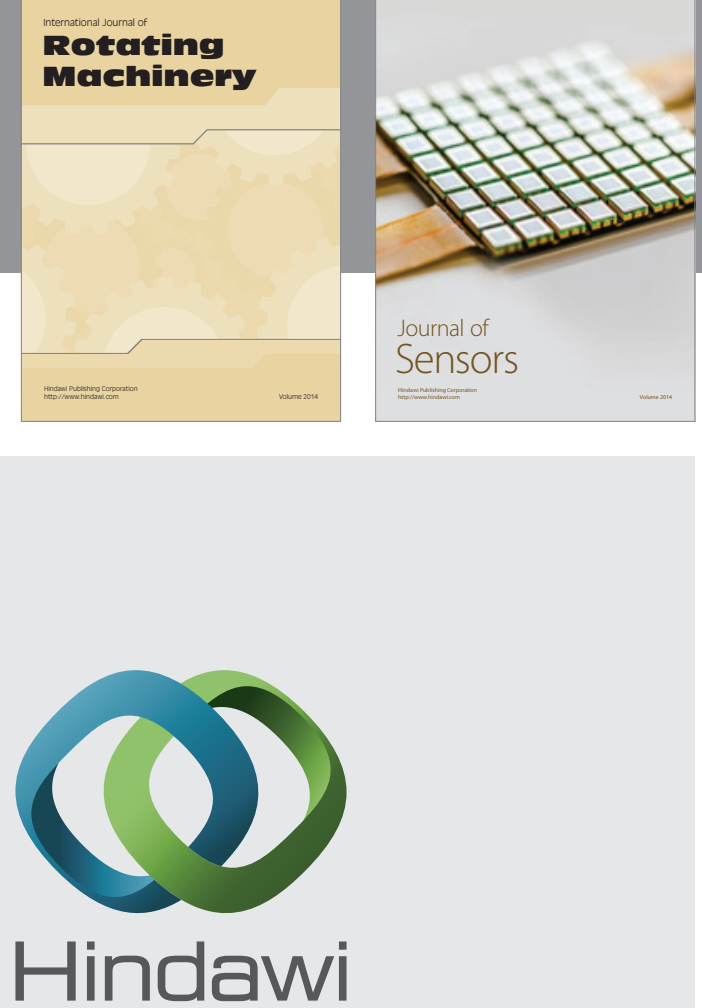

Submit your manuscripts at http://www.hindawi.com
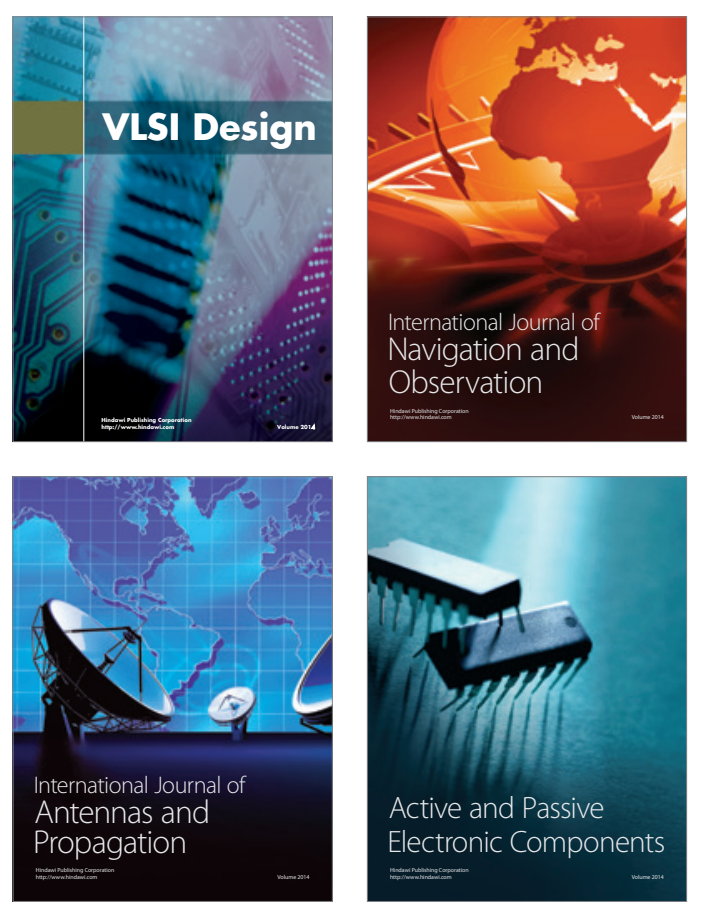
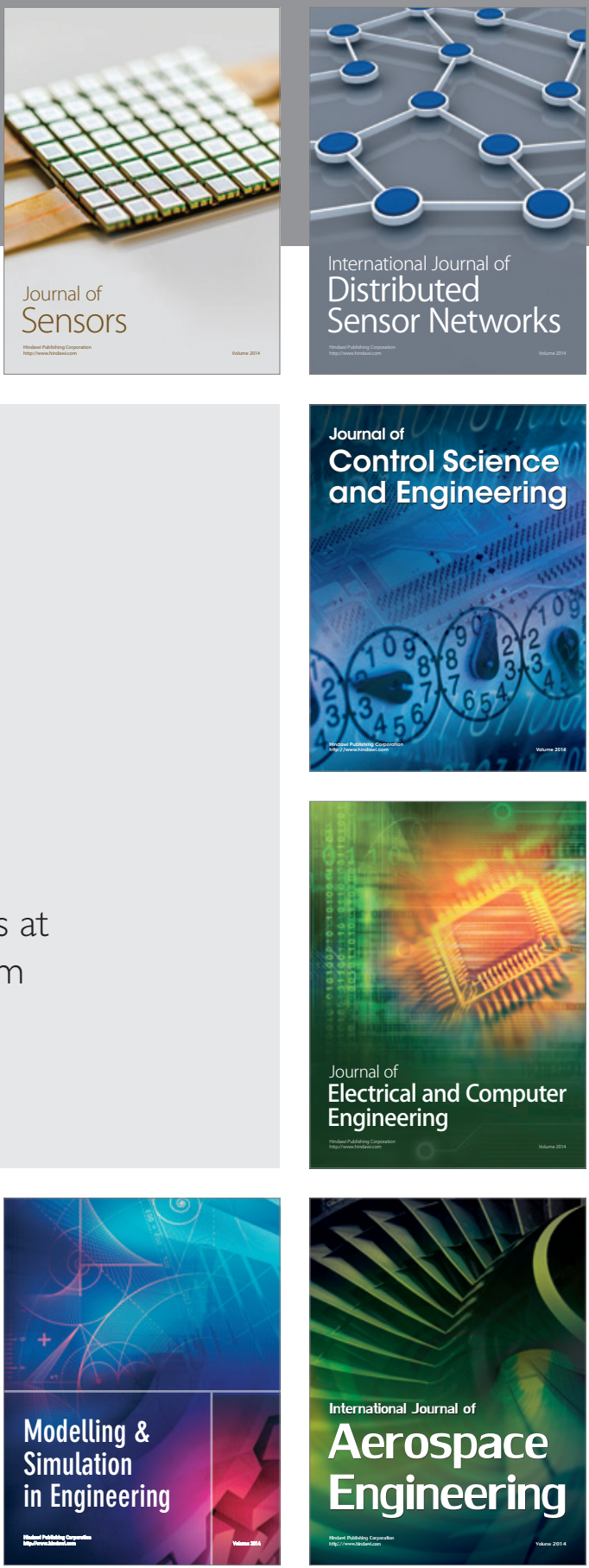

Journal of

Control Science

and Engineering
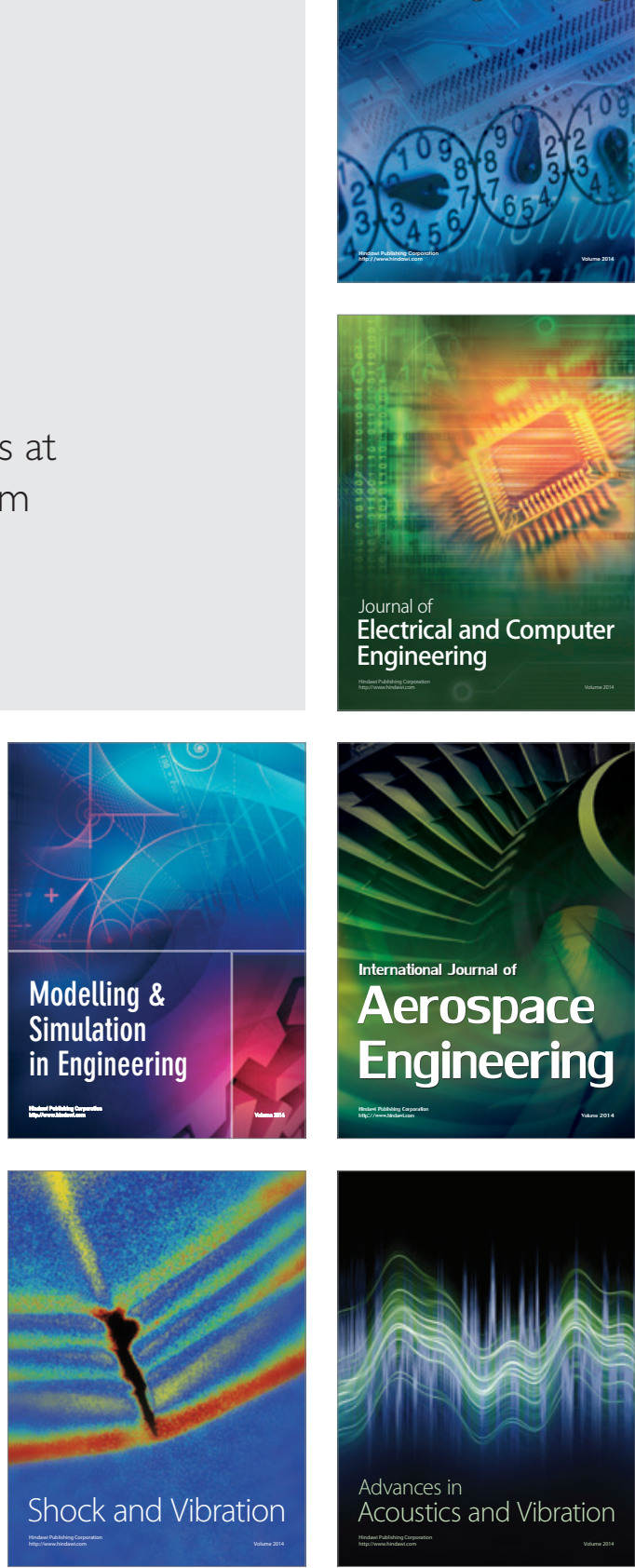